and the expression of TNF-a and VCAM-1 in ileum were observed by $\mathrm{H}$.E staining and immune chemical methods

Results 12 animals in each group, 9 in group NT, 10 in group SC and 9 in group PC were successfully resuscitated; all animals were on mechanical ventilation for 2 to $4 \mathrm{~h} \mathrm{5,6}$ and 8 animals in each group respectively survived to the end of the experiment. The temperatures of tympanic and peritoneal cavity of animals in group NT were maintained in normal range. The tympanic temperature of animals in group SC and PC was arrived target temperatures at $29 \pm 6.55$ mins and $62 \pm 8.27$ mins. During the stage of maintenance of hypothermia, the tympanic and peritoneal temperatures of animals in group SC were in range 33 to $35^{\circ} \mathrm{C}$, while the peritoneal temperatures of animals in group $\mathrm{PC}$ were in range 31 to $34^{\circ} \mathrm{C}, 1$ to $2^{\circ} \mathrm{C}$ lower than the tympanic temperature. The scores of histological injured of ileum were $1.43 \pm 0.53$ in group PC, $3.4 \pm 0.55$ in group NT and $3.17 \pm 0.41$ in group SC. The differences among them were significantly, PC vs SC, $\mathrm{p}<0.000$; PC vs NT, $\mathrm{p}<0.000$; while SC vs NT, $\mathrm{p}=0.30$. The expression of TNF-a in ileum was $9.98 \pm 1.79 \%$ in group NT, $5.87 \pm 1.43 \%$ in group SC and $3.78 \pm 0.53 \%$ in group PC, the differences among them were significantly. The phenomenon of the expression of VCAM-1 was little like the TNF-a, $3.78 \pm 0.53 \%$ in group PC was significantly from the $8.53 \pm 1.53 \%$ in group NT and $5.92 \pm 1.06 \%$ in group SC.

Conclusion The neotype peritoneal cooling can improve the injured of ileum mucous beside quickly induce hypothermia after ROSC in rabbits.

\section{e0224 MODEL OF CARDIAC ARREST IN RATS BY TRANSCUTANEOUS ELECTRICAL EPICARDIUM STIMULATION}

doi:10.1136/hrt.2010.208967.224

\begin{abstract}
${ }^{1}$ Lin Ji-yan, ' ${ }^{1}$ Li Xin, ${ }^{1}$ Li Hui, ' Wei Hong-yan, 'Liu Rong, ${ }^{1} H u$ Chun-lin, ${ }^{2}$ Dai Gang, ${ }^{1}$ Liao Xiao-xing. ${ }^{1}$ Department of Emergency Medicine, The First Affiliated Hospital of Sun Yat-Sen University, Guangdong, China; ${ }^{2}$ Key Lab on Assisted Circulation of Ministry of Health of Sun Yat-Sen University, Guangzhou, China
\end{abstract}

Objective To establish a new model of Cardiac Arrest (CA) in rats by transcutaneous electrical epicardium stimulation.

Methods Two acupuncture needles connected to the anode and cathode of a stimulator were transcutaneously inserted into the epicardium as electrodes. The stimulating current was steered to the epicardium and the stimulation was maintained for 3 min to induce CA. Cardiopulmonary resuscitation (CPR) was performed at 6 min after a period of nonintervention.

Results The success rate of induction was $12 / 20$ at the current intensity of $1 \mathrm{~mA}$; and reached 20/20 when the current intensity was increased to $2 \mathrm{~mA}$. The average time from the electrical stimulation to CA induction was $5.10( \pm 2.81) \mathrm{s}$. When the electrical stimulation stopped, 18/20 rats had ventricular fibrillation and $2 / 20$ rats had pulseless electrical activity. CPR was performed for averagely $207.4( \pm 148.8) \mathrm{s}$. The restoration of spontaneous circulation was $20 / 20$. The death rate within $4 \mathrm{~h}$ after CA was $5 / 20$, and the $72-$ h survival rate was $10 / 20$. There were only two cases of complications, a minor muscle contraction and a minor lung lobe injury.

Conclusion The model of CA in rats induced by transcutaneous electrical epicardium stimulation is a stable model that requires lowintensity current and has fewer complications.

\section{e0225 HYDROGEN SULFIDE INHABITS NEURONS APOPTOSIS IN RATS AFTER CARDIOPULMONARY RESUSCITATION}

doi:10.1136/hrt.2010.208967.225

${ }^{1}$ Xiao-xing Liao, ${ }^{1} \mathrm{Ji}$-yan Lin, ${ }^{1} \mathrm{H}$ ong-yan Wei, ${ }^{1} \mathrm{Hui}$ Li, ${ }^{1} \mathrm{Xin} \mathrm{Li}$, ${ }^{1}$ Rong Liu, ${ }^{1}$ Chun-lin Hu, ${ }^{1}$ Guo-qing Huang, ${ }^{2}$ Gang Dai. ${ }^{1}$ Department of Emergency, the First Affiliated Hospital of
Sun Yat-Sen University, Guangzhou, China; ${ }^{2}$ Key Lab on Assisted Circulation of Ministry of Health of Sun Yat-Sen University, Guangzhou, China

Objective To investigate the effects of hydrogen sulfide $\left(\mathrm{H}_{2} \mathrm{~S}\right)$ on brain injury after cardiopulmonary resuscitation (CPR) in rats by examining neurons apoptosis.

Methods The 40 male SD rats were randomly divided into experimental and control groups equally. In control group, CPR was performed with Utstein mode at 6 min after CA. On this basis, sodium hydrosulfide was administrated to the rats after restoration of spontaneous circulation in experimental group. On seventh day after CPR, neurons apoptosis was examined using terminal deoxynucleotidyl transferase mediated dUTP biotin nick end labelling (TUNEL) staining and the expression of caspase-3 was detected by the immunohistochemical strepto avidin biotinperoxidase complex (SABC) method in cortex, hippocampus CA1 region and cerebellum of the rats.

Results 1 . There were 12 and 10 rats completed the experiment in the experimental and control group respectively. Their fate between the two groups was no significant difference $\left(\chi^{2}=0.404, p=0.376\right) .2$. On seventh day after $\mathrm{CPR}$, The serum concentrations of $\mathrm{H}_{2} \mathrm{~S}$ was $9.12 \pm 3.17 \mu \mathrm{mol} / 1$ in the experimental group and the contrast was $3.72 \pm 1.05 \mu \mathrm{mol} / \mathrm{l}$, the difference between the two groups had statistic significance $(t=5.136, p=0.000)$. 3. Compared with the control group, the experimental group's neurons apoptosis index and the sum of integrated optical density (IOD) of caspase- 3 in cortex, hippocampus CA1 region and cerebellum were obviously reduced $(p<0.05)$.

Conclusion After CPR, $\mathrm{H}_{2} \mathrm{~S}$ can inhabit neurons apoptosis and its mechanism may be through caspase- 3 pathway. It may play a role in the treatment of the brain injury after CA.

\section{e0226 EFFECTS OF BONE MARROW MESENCHYMAL STEM CELLS ON ELECTROPHYSIOLOGICAL FUNCTION IN RATS WITH MYOCARDIAL INFARCTION}

doi:10.1136/hrt.2010.208967.226

Wang Deguo, Zhang Fengxiang, Shen Wenzhi, Chen Hongwu, Yang Bing, Chen Minglong, Cao Kejiang. The First Affiliated Hospital of Nanjing Medical University, Nanjing, Jiangsu, China

Objective Concerns that intramyocardial delivery of immature cells could cause potentially life-threatening ventricular arrhythmias have been repeatedly raised. The aim of this study is to investigate the electrophysiological and arrhythmogenic effects for MSCs therapy in AMI.

Methods GFP tagged MSCs were injected into a murine heart with left anterior descending (LAD) ligation. Two weeks after transplantation, effective refractory period (ERP), ventricular arrhythmias (VAs) inducibility and ventricular fibrillation threshold (VFT) were assessed by programmed electrical stimulation (PES), respectively. Epicardial monophasic action potential (MAP) recordings were obtained from infarcted border zone (IBZ) and none infarcted zone (NIZ) of left ventricular epicardium for calculation action potential duration (APD) and activation time (AT). Immunofluorescence and immunoblots were used to determine the expression and distribution of $\mathrm{Cx} 43$, collagen I and Kv4.2.

Results PES showed a significant reduced VTs, raised VFT and VERP in MSCs treated rats compared to PBS treated animals. MSCs implantation led to markedly longer APD and shorter AT in IBZ than PBS treated hearts. Histological study revealed that fibrotic area and collagen deposition in infarcted region were significantly lower in MI-MSCs group than in MI-PBS group. Abnormal alterations of $\mathrm{Cx} 43$ including reduction and lateralisation were significantly attenuated by MSCs treatment. Inhibition of Kv4.2 expression was partly ameliorated by MSCs therapy. 